\title{
New Equivalents of the Axiom of Choice and Consequences
}

\author{
Milan R. TAsković
}

\begin{abstract}
This paper continues the study of the Axiom of Choice by E. Z e r m el o [Neuer Beweis für die Möglichkeit einer Wohlordung, Math. Annalen, 65 (1908), 107-128; translated in van Heijenoort 1967, 183-198], and by M. Ta s k ov i ć [The axiom of choice, fixed point theorems, and inductive ordered sets, Proc. Amer. Math. Soc., 116 (1992), 897-904].
\end{abstract}

\section{INTRODUCTION AND ORIGINS}

Well known is that equivalents of the Axiom of Choice appear frequently in almost all branches of mathematics and nature in a large variety of different forms. In 1908 E. Zermelo proposed main version of the Axiom of Choice. This is the connection and with some conversations with Erhard Schmidt.

Apparently the first specific reference to the axiom of choice was given in a paper by G. Peano in 1890. In proving an existence theorem for ordinary differential equations, he ran across a situation in which such a statement is needed.

In 1886 Peano published a new demonstration of the theorem, due to A. Cauchy, that the differential equation

$$
y^{\prime}=f(x, y), \quad y\left(x_{0}\right)=t_{0},
$$

has a unique solution. Here Peano weakened Cauchy's hypotheses to require only that $f(x, y)$ be continuous. Four years later Peano returned to this theorem and generalized his proof to finite systems of first-order equations.

Beppo Levi in 1902, while discussing the statement that the union of a disjoint set $S$ of nonempty sets has a cardinal number greater than or equal to the cardinal number of $S$, remarked that its proof depended on the possibility of selecting a single member from each element of $S$. Others, including Georg Cantor, had used the principle earlier, but did not mention it specifically.

2000 Mathematics Subject Classification. Primary: 47H10; Secondary: 54H25. 
In 1892 R. Bettazzi, who had just become Peano's colleague at the Military Academy in Turin, published an article on discontinuous real functions - with terminology of infinite many arbitrary choices.

We notice that the Axiom of Choice is main spring (origin) for the fixed point theory. In this sense, the fixed point problem for a given mapping $f \mid P$ is very easy to formulate: the question is whether some $\xi \in P$ satisfies $f(\xi)=$ $\xi$. Many problems are reducible to the existence of fixpoints of certain mappings. The question remains whether some statement (of the axiom of choice type) could be equivalently expressed in the fixpoint language as well. The answer is affirmative. In this sense, the equation for $x \in P$ in the following form

$$
\sup \{x, f(x)\}=x \quad\left(\text { or } \sup \left\{x, f^{2}(x)\right\}=x\right)
$$

for a given map $f \mid P$ and for a nonempty partially ordered set $P$ is a key object for new equivalents of the Axiom of Choice.

In this paper we prove some new equivalents of the Axiom of Choice in connection with (Eq). These statements are of fixed point type theorems and fixed apex type theorems. Applications in fixed point theory are considered.

Call a poset (=partially ordered set) $P$ inductive (chain complete) when every nonempty chain in $P$ has an upper bound (least upper bound, i.e., supremum) in $P$. Also, call a poset $P$ quasi-inductive (quasi-chain complete) when every nonempty well ordered chain has an upper bound (supremum) in $P$.

In [20] we consider the concept of fixed apices for the mapping $f$ of a poset $P$ into itself. A map $f$ of a partially ordered set $P$ to itself has a fixed apex $u \in P$ if for $u \in P$ there is $v \in P$ such that $f(u)=v$ and $f(v)=u$.

Fixed points are clearly fixed apices and the set of all fixed points can be a proper subset of the set of fixed apices.

On the other hand, $f$ has a fixed apex if and only if $f^{2}:=f(f)$ has a fixed point. Indeed, for if $f$ has a fixed apex $u \in P$, then $u=f(v)$ and $v=f(u)$, so $f^{2}$ has a fixed point. If the equation $x=f^{2}(x)$ has a solution $\xi=f^{2}(\xi)$ for some $\xi \in P$, then $f$ has fixed apices $\xi, f(\xi) \in P$ because $\xi=f^{2}(\xi)$ and $f(\xi)=f(\xi)$.

\section{MAin Results AND APPLICATIONS}

We are now in a position to formulate the following results. Let $P$ be a partially ordered set and $f$ a mapping from $P$ into $P$. For any $f: P \rightarrow P$ it is natural to consider the following set:

$$
\operatorname{Sub} f(P):=f(P) \cup\{a \in P \mid a=u b C \text { for some chain } C \text { in } f(P)\},
$$

where $u b C$ is an upper bound of $C$. This set is first time considered in [19]. 
Theorem 1 (Axiom of Choice for Points). Let $P$ be a set partially ordered by an ordering relation $\preccurlyeq$. Then the following statements are equivalent:

(a) (Zorn's lemma). Let $P$ be an inductive partially ordered set. Then $P$ has at least one maximal element.

(b) Let $P$ be an inductive partially ordered set and $f$ a mapping from $P$ into $P$ such that

$$
x \preccurlyeq \sup \{x, f(x)\} \quad \text { for all } \quad x \in \operatorname{Sub} f(P),
$$

then the function $\varphi(x):=\sup \{x, f(x)\}$ has at least one fixed point. If for all $a, b \in P$ the following condition holds in the form as

$$
\sup \{a, b\}=a \quad \text { implies } \quad a=b,
$$

then the mapping $f$ has at least one fixed point. (Also, the dually statement of this statement holds).

(c) Let $P$ be an inductive partially ordered set and $f$ a mapping from $P$ into $P$ such that

$$
x \preccurlyeq f(x) \text { for all } x \in \operatorname{Sub} f(P),
$$

then $f$ has at least one fixed point. (Also, the dually statement of this statement holds).

Proof. From [22] (Theorem 1, p. 898), (a) is equivalent to the (c). Thus, we need only show that (a) implies (b) and that (b) implies (c).

(a) implies (b). By Zorn's lemma there exists a maximal element $z \in P$, i.e., Sub $f(P)$ has a maximal element $z \in \operatorname{Sub} f(P)$. From condition (M) we have $z \preccurlyeq \sup \{z, f(z)\}=z$, and, because $z$ is a maximal element of set Sub $f(P), \sup \{z, f(z)\} \preccurlyeq z$. Hence, $\varphi(z):=\sup \{z, f(z)\}=z$, i.e., $\varphi$ has a fixed point. Applying condition (A) to the equality $\varphi(z)=z$ we obtain $f(z)=z$. Thus $f$ has fixed point.

(b) implies (c). From (N) we have $x \preccurlyeq f(x) \preccurlyeq \sup \{x, f(x)\}$ for all $x \in \operatorname{Sub} f(P)$. Since (M) holds, it follows from (b) that $\varphi(x):=\sup \{x, f(x)\}$ has a fixed point $\xi \in \operatorname{Sub} f(P)$. Thus, and from (N), the condition (A) holds for the points $\xi$ and $f(\xi)$. Applying (b) to this fact we obtain that $f$ has a fixed point. The proof is complete.

Annotations. The proof that (c) implies (a) may be found on [22] (p. 898) and on [19] (p. 85). In connection with this we give in the next a different proof of the preceding:

(c) implies (a). (Proof sketch). Suppose that (a) is false. Let $L_{0}$ be the family of all nonempty linearly ordered subsets of $P$ and let $L=L_{0} \cup\{\varnothing\}$. The family $L$ is partially ordered by the inclusion relation $\subset$ between subsets of $P$. For each $A \in L_{0}$ the set

$$
A_{U}=\{x \in P: x \quad \text { is an upper bound for } A \text { and } x \notin A\}
$$


is nonempty because if $x$ is an upper bound for $A$ and $y \in P$ is such that $x \preccurlyeq y$ and $x \neq y$ then $u \in U_{A}$. Let $U_{\varnothing}=\left\{x_{0}\right\}$, where $x_{0}$ is an arbitrary element of $P$.

Let $f$ be a function defined on $L$ such that $f(A)=A \cup\left\{g\left(U_{A}\right)\right\}$ for all $A \in L$, where $g$ is the identity mapping $g(x)=x$ with domain $\left\{U_{A}: A \in L\right\}$.

Now, $L$ satisfies the condition of inductiveness of (c). By definition of $f$ we have $A \subset f(A)$ so that condition (N) of (c) is satisfied; but $f$ has no fixed point, i.e., we have a contradiction. This completes the proof.

In connection with the preceding statement, we notice that statement (b) of Theorem 1 we can give in the following form: Let $P$ be an inductive poset and $f$ a mapping from $P$ into itself such that $(\mathrm{M})$, then the equation in the form as

$$
\sup \{x, f(x)\}=x
$$

has at least one solution on the set $P$. If condition (A) holds, then the equation $x=f(x)$ has at least one solution on the set $P$.

Theorem 2 (Axiom of Choice for Apices). Let $P$ be a set partially ordered by an ordering relation $\preccurlyeq$. Then the following statements are equivalent:

(a) (Zorn's lemma). Let $P$ be an inductive partially ordered set. Then $P$ has at least one maximal element.

(b) Let $P$ be an inductive partially ordered set and $f$ a mapping from $P$ into $P$ such that

$$
x \preccurlyeq \sup \left\{x, f^{2}(x)\right\} \quad \text { for all } \quad x \in \operatorname{Sub} f(P),
$$

then the function $\psi(x):=\sup \left\{x, f^{2}(x)\right\}$ has at least one fixed point. If condition (A) holds, then the mapping $f$ has a fixed apex. (Also, the dually statement of this statement holds).

(c) Let $P$ be an inductive partially ordered set and $f$ a mapping from $P$ into $P$ such that

$$
x \preccurlyeq f^{2}(x) \text { for all } x \in \operatorname{Sub} f(P),
$$

then $f$ has at least one fixed apex. (Also, the dually statement of this statement holds).

Proof. From [22] (Theorem 1, p. 898), (a) is equivalent to the (c). Thus, we need only show that (a) implies (b) and that (b) implies (c). First, (a) implies (b). By Zorn's lemma there exists a maximal element $z \in P$, i.e., Sub $f(P)$ has a maximal element $z \in \operatorname{Sub} f(P)$. From condition $(\mathrm{R})$ we have $z \preccurlyeq \sup \left\{z, f^{2}(z)\right\}$ and, because $z$ is a maximal element of set $\operatorname{Sub} f(P), \sup \left\{z, f^{2}(z)\right\} \preccurlyeq z$. Hence, $\psi(z):=\sup \left\{z, f^{2}(z)\right\}=z$, i.e., $\psi$ has a fixed point. Applying condition (A) to the equality $\psi(z)=z$ we obtain $f^{2}(z)=z$. This means, from the initial facts for apices, that $f$ has fixed apices $z, f(z) \in P$ because $z=f^{2}(z)$ and $f(z)=f(z)$.

(b) implies (c). From (T) we have $x \preccurlyeq f^{2}(x) \preccurlyeq \sup \left\{x, f^{2}(x)\right\}$ for all $x \in$ Sub $f(P)$. Since $(\mathrm{R})$ holds, it follows from (b) that $\psi(x):=\sup \left\{x, f^{2}(x)\right\}$ 
has a fixed point $\eta \in \operatorname{Sub} f(P)$. Thus, and from (T), the condition (A) holds for the points $\eta$ and $f^{2}(\eta)$. Applying (b) to this fact we obtain that $f$ has a fixed apex. The proof is complete.

Annotations. In connection with the preceding two statements we notice that as proved in [22] the following statements are equivalent: Zorn's lemma and the following statement.

Also, we notice that statement (b) of Theorem 2 we can give in the following profitable form for applications: Let $P$ be an inductive poset and $f$ a mapping from $P$ into itself such that $(\mathrm{R})$, then the equation in the form as

$$
\sup \left\{x, f^{2}(x)\right\}=x
$$

has at least one solution on the set $P$. If condition (A) holds, then the equation $x=f^{2}(x)$ has at least one solution on the set $P$.

Theorem 3 (Zermelo [24]). Let $P$ be a chain complete partially ordered set and $f$ a mapping from $P$ into itself such that:

(a) there is an element $\theta \in P$ with $\theta \preccurlyeq x$ for all $x \in P$;

(b) $x \preccurlyeq f(x)$ for all $x \in P$;

(c) if $x, y \in P$ and $x \preccurlyeq y \preccurlyeq f(x)$ then either $x=y$ or $f(x) \preccurlyeq f(y)$.

Then $f$ has a fixed point.

A proof that Zorn's lemma is equivalent to Theorem 3 may be found first time in the author's Thesis [19]. For further facts of this see [23].

Our next purpose is to prove some results about partial order relations, now usually called Zorn's lemma, which has far-reaching consequences in several branches of mathematics. We give a strong form of Zorn's lemma due to Bourbaki, for well-ordered $(:=w o)$ chains.

Lemma 1 (Bourbaki [4]). Let $P$ be a quasi-inductive partially ordered set. Then $P$ has at least one maximal element.

Let $P$ be a partially ordered set and $f$ a mapping from $P$ into $P$. For any $f: P \rightarrow P$ it is natural to consider the following set of the form as

$$
\operatorname{Sub}[f(P)):=f(P) \cup\{a \in P: a=u b C \text { for some wo chain } C \text { in } f(P)\},
$$

where $u b C$ is an upper bound of well-ordered chain in $C$. This set is first time considered in [22].

In the next, we consider elements of the apices equation for $x \in P$ in the following form

$$
\sup \left\{x, f^{2}(x)\right\}=x
$$

for a given map $f \mid P$ and for a nonempty partially ordered set $P$. Also, this is a key object for new equivalents of the Axiom of Choice. 
Lemma 2 (Lemma of Equation Apices). Let $P$ be a quasi-inductive partially ordered set and $f$ a mapping from $P$ into $P$ such that

$$
x \preccurlyeq \sup \left\{x, f^{2}(x)\right\} \quad \text { for all } \quad x \in \operatorname{Sub}[f(P)) \text {, }
$$

then the function $\psi(x):=\sup \left\{x, f^{2}(x)\right\}$ has a fixed point. If the condition (A) holds, then the mapping $f$ has a fixed apex. (Also, the dually statement of this statement holds).

Proof. By Lemma 1 there exists a maximal element $z \in P$, i.e., $\operatorname{Sub}[f(P))$ has a maximal element $z \in \operatorname{Sub}[f(P))$. From condition (Rw) we have $z \preccurlyeq \sup \left\{z, f^{2}(z)\right\}$ and, because $z$ is a maximal element of $\operatorname{Sub}[f(P))$, we have $\sup \left\{z, f^{2}(z)\right\} \preccurlyeq z$. Hence, $\psi(z)=z$; so applying condition (A) to this fact, from the initial facts for apices, we obtain that $f$ has a fixed apex. The proof is complete.

The lemmas help to obtain the following result. We show later that the existence of a fixed apex for each mapping from a poset into itself that satisfies $(\mathrm{Rw})$ is a necessary and sufficient condition for the poset to be quasi-inductive.

Theorem 4. If $P$ is a poset such that $(\mathrm{A})$ and that every $f: P \rightarrow P$ that satisfies $(\mathrm{Rw})$ has a fixed apex, then $P$ is quasi-inductive. (The Axiom of Choice is not used in this proof).

Proof. We have to show that under the assumption that the poset $P$ is not quasi-inductive there exists a mapping $f$ on $P$ to $P$ with condition $(\mathrm{Rw})$ and without fixed apices.

Suppose $P$ is not quasi-inductive. Then there exists a well-ordered chain $C$ in $P$ that has no upper bound. Let $U$ be a chain cofinal with $C$ such that

$$
U:=\left\{x \in C \mid x_{0} \preccurlyeq x\right\}, \quad x_{0}:=\text { a fixed element of } C=\min U .
$$

Thus all elements of $U$ can be arranged in a sequence, i.e., one can show that there exists a strictly increasing sequence $\left\{x_{\alpha}\right\}$ in $U$ such that its upper bound does not exist. We define a mapping $f$ from $P$ into itself by

$$
f(x)=\left\{\begin{array}{l}
x_{\alpha+1} \text { if } x=x_{\alpha} \in U, \\
x_{0}:=\min U, \text { if } x \notin U,
\end{array}\right.
$$

where $x_{\alpha} \preccurlyeq x_{\alpha+1}\left(x_{\alpha} \neq x_{\alpha+1}\right)$ for any $\alpha \prec w$, and where $w$ is any ordinal. Now, for any $x \in U(\supset \operatorname{Sub}[f(P)))$ we have $x \preccurlyeq f^{2}(x) \preccurlyeq \sup \left\{x, f^{2}(x)\right\}$, i.e., $x=x_{\alpha} \preccurlyeq x_{\alpha+2}=f\left(x_{\alpha+1}\right)=f\left(f\left(x_{\alpha}\right)\right)=f^{2}\left(x_{\alpha}\right)=f^{2}(x) \preccurlyeq \sup \left\{x, f^{2}(x)\right\}$ for $\alpha \prec w$, so $f$ satisfies ( $\mathrm{Rw})$ and does not have a fixed apex. The proof is complete.

The following result of Bourbaki [4] allows us to prove the basic fixpoint statement for chain complete posets. Also see and an essential result by Amann [2]. 
Lemma 3. Let $P$ be a chain complete partially ordered set and $f: P \rightarrow P$ a map such that $x \preccurlyeq f(x)$ for all $x \in P$. Then $f$ has a fixed point.

Our next statement extends this lemma with chain complete posets to quasi-inductive partially ordered sets in the following sense.

Lemma 4 (Lemma of Equation Points). Let $P$ be a quasi-inductive partially ordered set and $f: P \rightarrow P$ a map such that

$$
x \preccurlyeq \sup \{x, f(x)\} \quad \text { for all } \quad x \in \operatorname{Sub}[f(P)) \text {, }
$$

then the function $\varphi(x):=\sup \{x, f(x)\}$ has at least one fixed point. If condition (A) holds, then the mapping $f$ has a fixed point. (Also, the dually statement of this statement holds).

The proof of this statement is a totally analogous with the proof of Lemma 2. A brief proof of this statement based on Bourbaki's lemma may be found in $[22]$.

We say that $P$ has the equation-general fixed point property if (A) and if every map $f$ of $P$ into itself with condition $(\mathrm{Mw})$ has a fixed point. Analogously, $P$ is said to have the equation-general fixed apex property if (A) and if every map $f$ of $P$ into itself with condition $(\mathrm{Rw})$ has a fixed apex.

Theorem 5. Let $P$ be a partially ordered set. Then the following statements are equivalent:

(a) $P$ is quasi-inductive;

(b) $P$ has the equation-general fixed apex property;

(c) $P$ has the equation-general fixed point property.

Proof. From Theorem 4 and Lemma 2, (a) is equivalent to (b). Lemma 4 implies that (c) is a consequence of (a). Thus, we need only show that (c) implies (a).

Suppose $P$ is not quasi-inductive. Then there is a chain $C$ in $P$ that does not have an upper bound. Let $U$ be an ordered chain cofinal with $C$. Define a mapping $f$ from $P$ into itself by (1). Then $f$ is well defined and for any $x \in \operatorname{Sub}[f(P))$ we have $x \preccurlyeq f(x) \preccurlyeq \sup \{x, f(x)\}$, i.e., $x=x_{\alpha} \preccurlyeq x_{\alpha+1}=$ $f\left(x_{\alpha}\right)=f(x) \preccurlyeq \sup \{x, f(x)\}$. Thus, $f$ satisfies (Mw) and does not have a fixed point.

Theorem 6. If $P$ is a poset such that (A) and that every mapping $f: P \rightarrow$ $P$ that satisfies $(\mathrm{R})$ has a fixed apex, then $P$ is quasi-inductive. (The Axiom of Choice is not used in the proof).

The proof of this statement is analogous to the proof of the preceding statements. A brief similarly proof of this statement may be found in [23].

As indicated in [20], $P$ is said to have the equation fixed point property if (A) and if every map $f$ of $P$ into itself with the condition (M) has a fixed 
point. Analogously, $P$ is said to have the equation fixed apex property if (A) and if every map $f$ of $P$ into itself with the condition (R) has a fixed apex. The following statement summarizes things in [22] and in preceding statements.

Theorem 7. Let $P$ be a partially ordered set. Then the following statements are equivalent: (a) $P$ is inductive, (b) $P$ is quasi-inductive, (c) $P$ has the equation fixed apex property, (d) $P$ has the equation fixed point property, (e) $P$ has the equation-general fixed apex property, (f) $P$ has the equationgeneral fixed point property.

We notice, as an immediate application of the preceding statements (equivalents of inductiveness and quasi-inductiveness), that Zorn's lemma and Bourlaki's lemma are equivalent. Analogously, Bourbaki's lemma, Lemma of Equation Apices, and Lemma of Equation Points are equivalent. Also, we have the following statement as a consequence.

Let $P$ be a partially ordered set and $f$ a mapping from $P$ into $P$. For any $f: P \rightarrow P$ it is natural to consider the set

$$
\overline{f(P)}:=f(P) \cup\{a \in P \mid a=\sup C \text { for some chain } C \text { in } f(P)\} .
$$

Theorem 8. Let $P$ be a set partially ordered by an ordering relation $\preccurlyeq$. Then the following statements are equivalent:

(a) (Bourbaki's lemma). Let $P$ be a quasi-inductive partially ordered set, then $P$ has at least one maximal element.

(b) Let $P$ be a quasi-chain complete partially ordered set. Then $P$ has at least one maximal element.

(c) Let $P$ be a chain complete partially ordered set and $f$ a mapping from $P$ into itself such that

$$
x \preccurlyeq \sup \left\{x, f^{2}(x)\right\} \quad \text { for all } x \in \overline{f(P)},
$$

then the function $\psi(x):=\sup \left\{x, f^{2}(x)\right\}$ has a fixed point. If the condition (A) holds, then the mapping $f$ has a fixed apex. (Also, the dually statement of this statement holds).

(d) Let $P$ be a chain complete partially ordered set and $f$ a mapping from $P$ into itself such that

$$
x \preccurlyeq \sup \{x, f(x)\} \quad \text { for all } \quad x \in \overline{f(P)},
$$

then the function $\varphi:=\sup \{x, f(x)\}$ has a fixed point. If the condition (A) holds, then the mapping $f$ has a fixed point. (Also, the dually statement of this statement holds).

Based on Theorem 8 the above facts, we can prove the following statement as a localization of the preceding statement.

Theorem 9 (Local forms). Let $P$ be a partially ordered set with ordering relation $\preccurlyeq$. Then the following statements are equivalent: 
(a) (Zorn's lemma). Let $P$ be an inductive partially ordered set. Then $P$ has at least one maximal element.

(b) Let $P$ be a quasi-chain complete partially ordered set and $f$ an increasing mapping from $P$ into $P$ such that

$$
a \preccurlyeq \sup \left\{a, f^{2}(a)\right\} \quad \text { for some } a \in P,
$$

then the function $\psi(x):=\sup \left\{x, f^{2}(x)\right\}$ has a fixed point. If the condition (A) holds, then the mapping $f$ has a fixed apex. (Also, the dually statement of this statement holds).

(c) Let $P$ be a quasi-chain complete partially ordered set and $f$ an increasing mapping from $P$ into $P$ such that

$$
a \preccurlyeq \sup \{a, f(a)\} \quad \text { for some } a \in P,
$$

then the function $\varphi(x):=\sup \{x, f(x)\}$ has a fixed point. If the condition (A) holds, then the mapping $f$ has a fixed point. (Also, the dually statement of this statement holds).

Proof. (a) implies (b). Consider the subset $A$ of $P$ given by $A:=\{x \in P$ : $x \preccurlyeq \sup \left\{x, f^{2}(x)\right\}$. From the given condition we see that $A$ is nonempty. Since $x \preccurlyeq \sup \left\{x, f^{2}(x)\right\}$ implies $f(x) \preccurlyeq \sup \left\{f(x), f^{2}(f(x))\right\}$, and we therefore have that $f$ maps $A$ into $A$. Next, let $C$ be a nonempty well-ordered chain of $A$ and $\xi$ a least upper bound of $C$. Also, since $x \preccurlyeq \xi$ for every $x \in C$, it follows that $f^{2}(x) \preccurlyeq f^{2}(\xi)$ for every $x \in C$. However, since $C$ is a subset of $A$, it follows that $x \preccurlyeq \sup \left\{x, f^{2}(x)\right\} \preccurlyeq \sup \left\{\xi, f^{2}(\xi)\right\}$ for every $x \in C$. So $\sup \left\{\xi, f^{2}(\xi)\right\}$ is an upper bound of $C$ and, consequently, $\xi \preccurlyeq \sup \left\{\xi, f^{2}(\xi)\right\}$. It then follows that $A$ is a nonempty poset with the property that each nonempty well-ordered chain of $A$ has an upper bound, i.e., $A$ is a quasi-inductive set and $f$ maps $A$ into $A$; thus, according to (b) of Theorem 2, we see that $f$ has a fixed apex, as described.

The proofs for (b) implies (c) and (c) implies (a) are analogous to the proofs of the statements (b) implies (c) and (c) implies (a) of Theorems 1 and 2 .

An immediate consequence of the preceding Lemma of Equation Points and its proof is the following equivalent form of the Axiom of Choice.

Theorem 10. Let $\mathcal{F}$ be a family of mappings of a partially ordered set $P$ into itself with ordering $\preccurlyeq$ such that

$$
x \preccurlyeq \sup \{x, f(x)\} \quad \text { for all } x \in P \text {, all } f \in \mathcal{F} \text {; }
$$

and if $P$ is a quasi-inductive poset with the condition (A), then the family $\mathcal{F}$ has a common fixed point for all $f \in \mathcal{F}$.

On the other hand, an immediate consequence of the above Lemma of Equation Apices and its proof is the following equivalent form of the Axiom of Choice, also. 
Theorem 11. Let $\mathcal{F}$ be a family of mappings of a partially ordered set $P$ into itself with ordering $\preccurlyeq$ such that

$$
x \preccurlyeq \sup \left\{x, f^{2}(x)\right\} \quad \text { for all } x \in P \text {, all } f \in \mathcal{F} \text {; }
$$

and if $P$ is a quasi-inductive poset with the condition (A), then the family $\mathcal{F}$ has a common fixed apex for all $f \in \mathcal{F}$.

The proof of this statement is analogous to the proof of the preceding statement. A brief proof a similarly statement may be found in [22].

Annotations. In concluding, we note that the fixed apex theorems can easily be generalized from $f^{2}$ to $f^{n}:=f\left(f^{n-1}\right)$ for $n \geq 2$.

The conception of apices can be broadened from $f^{2}$ to $f^{n}$ for some positive integer $n \geq 2$. The points $u_{1}, \ldots, u_{n} \in P$ are called fixed apices of $f: P \rightarrow P$ if

$$
u_{1}=f\left(u_{2}\right), u_{2}=f\left(u_{3}\right), \ldots, u_{n-1}=f\left(u_{n}\right), u_{n}=f\left(u_{1}\right)
$$

or if

$$
u_{2}=f\left(u_{1}\right), u_{3}=f\left(u_{2}\right), \ldots, u_{n}=f\left(u_{n-1}\right), u_{1}=f\left(u_{n}\right)
$$

for some positive integer $n \geq 2$. For this conception of apices also see [22].

We notice that the map $f$ has fixed apices $u_{1}, \ldots, u_{n} \in P$ if and only if $f^{n}$ (for some $n \geq 2$ ) has a fixed point.

Indeed, for if $f$ has fixed apices $u_{1}, \ldots, u_{n} \in P$ then $u_{1}=f\left(u_{2}\right)=$ $f^{2}\left(u_{3}\right)=\cdots=f^{n}\left(u_{1}\right)$ or $u_{n}=f^{n}\left(u_{1}\right)=f\left(u_{n-1}\right)=\cdots=f^{n-1}\left(u_{1}\right)=$ $f^{n}\left(u_{n}\right)$, so $f^{n}$ has a fixed point. On the other hand, if the equation $x=$ $f^{n}(x)$ has a solution $\xi=f^{n}(\xi)$ for some $\xi \in P$, then $f$ has a fixed apices $\xi, f^{n-1}(\xi), f^{n-2}(\xi), \cdots, f(\xi)$ because $\xi=f^{n}(\xi), f^{n-1}(\xi)=f\left(f^{n-2}(\xi)\right), \cdots$, $f(\xi)=f(\xi)$.

It will be observed that all results for fixed apices from this paper as and from [22] hold for the generalizations of fixed apices given by (AL) and (AR). We state only the following basic result, which is also an equivalent form of the Axiom of Choice.

Lemma 5. Let $P$ be a quasi-inductive partially ordered set with ordering $\preccurlyeq$ and $f$ a mapping from $P$ into $P$ such that for some positive integer $n \geq 2$ is

$$
x \preccurlyeq \sup \left\{x, f^{n}(x)\right\} \quad \text { for all } \quad x \in \operatorname{Sub}[f(P)),
$$

then the function $\alpha(x):=\sup \left\{x, f^{n}(x)\right\}$ has a fixed point. If condition (A) holds, then the mapping $f$ has fixed apices. (Also, the dually statement of this statement holds).

The proof of this statement is analogous to the proof of Lemma 2. A brief proof of this statement for a similarly result may be found in [23].

Annotation. In connection with the preceding statement, we notice that Lemma 5 we can give in the following profitable form for applications: Let 
$P$ be a quasi-inductive poset and $f$ a mapping from $P$ into itself such that for some positive integer $n \geq 2$ is (Ra), then the equation in the form as

$$
\sup \left\{x, f^{n}(x)\right\}=x
$$

has at least one solution on the set $P$. If condition (A) holds, then the equation $x=f^{n}(x)$ has at least one solution on the set $P$.

\section{Foundation of the Fixed Point Theory}

We notice that, de facto, Lemma od Equation Points, i.e., Axiom of Choice is main geometrical spring for the Fixed Point Theory. In this sense we give some illustrations of equations (Eq), (Aq), statement (b) of Theorem 1 , statement (b) of Theorem 2, and the following equation for $x \in P$ and for some positive integer $n \geq 2$ in the form as

$$
\sup \left\{x, f^{n}(x)\right\}=x
$$

for a given map $f \mid P$ and for a nonempty partially ordered set $P$. This is also general a key object for new equivalents of the Axiom of Choice.

Illustration 1. (Tarski's theorem). Let $P$ be a poset. A self-mapping $f$ of $P$ into itself is called an isotone mapping if $x \preccurlyeq y$ implies $f(x) \preccurlyeq f(y)$. In the mid-1950's A. Tarski published a generalization of a former fixed point theorem for posets goes back to Tarski and Knaster in the form: Every complete lattice has the fixed point property, i.e., every isotone map $f \mid P$ of a complete lattice $P$ into itself has a fixed point. The Axiom of Choice is not used in the Tarski's proof.

But, we notice that Tarski's theorem is a consequence of the Axiom of Choice. Indeed, if for a complete lattice $P$ holds the Tarski's theorem, then $P$ is an inductive poset and the condition (M) of (b) in Theorem 1 holds. Since condition (A) also holds on complete lattice $P$ in the form that for an arbitrary isotone mapping $f: P \rightarrow P$ there exists an element $x \in P$ such that

$$
\sup \{x, f(x)\}=x \quad \text { implies } \quad x=f(x),
$$

hence applying statement (b) of Theorem 1 (i.e., Axiom of Choice) we obtain that $f$ has a fixed point. The proof is complete.

Annotations. From the preceding illustration we have that Tarski's theorem is a consequence of Axiom of Choice. This is a result of the fact that the equation for $x \in P$ and for given an arbitrary isotone mapping $f: P \rightarrow P$ in the form as

$$
\sup \{x, f(x)\}=x
$$

has at least one solution on a complete lattice $P$. But, very interesting, for given an arbitrary antitone mapping $f: P \rightarrow P$ the equation (Fp) has not always solution on a complete lattice $P$. For this see the following fact.

Illustration 2. (The equation $\sup \left\{x, f^{2}(x)\right\}=x$ ). Let $P$ be a poset. A self-mapping $f$ on $P$ into itself is called an antitone mapping if $x \preccurlyeq y$ implies 
$f(y) \preccurlyeq f(x)$. In [20] we have without the Axiom of Choice the following result of J. Klimeš: If $P$ is a complete lattice and if $f$ is an antitone mapping of $P$ into itself, then $f$ has a fixed apex.

We notice that this result of Klimeš [9] is a consequence of the Axiom of Choice. Indeed, if for a complete lattice $P$ holds the preceding result, then $P$ is an inductive poset and the condition $(\mathrm{R})$ of $(\mathrm{b})$ in Theorem 2 holds. Since in this case, from Illustration 1, the condition (A) holds because the equation in the form (Aq) has a solution, thus applying statement (b) of Theorem 2 (i.e., Axiom of Choice) we obtain that $f$ has a fixed apex. The proof is complete.

Illustration 3. (Brouwer's theorem). The most famous of all fixed point theorems is that of Brouwer which satisfies that: every continuous mapping of the closed unit ball in the Euclidean space $\mathbb{R}^{n}$ into itself has a fixed point.

A new proof for this result (without Axiom of Choice) may be found in [23]. But, we notice that Brouwer's theorem is a consequence of the Axiom of Choice, also.

Indeed, if $\leq$ well-ordered on $P:=\mathbb{R}^{n}$ (from the well-ordering theorem), then every chain in $P$ has a lower bound and dually condition of $(\mathrm{M})$ for (b) in Theorem 1 holds. Since the dually condition of (A) also holds, thus applying the dually statement of (b) in Theorem 1 (i.e., Axiom of Choice) we obtain that $f$ has a fixed point. The proof is complete.

Annotations. In the preceding context of this illustration we obtain the following result: If $C$ is a nonempty convex compact subset of $\mathbb{R}^{n}$ and if $f: C \rightarrow C$ is a continuous mapping, then the equation (Fp) has a solution on $C$.

Illustration 4. (Schauder's theorem). Schauder's theorem is a generalization of Brouwer's theorem to infinite dimensional normed linear spaces. Schauder's theorem states that: every continuous mapping of a compact convex subset of a normed linear space into itself has a fixed point.

Also, Schauder's theorem is a consequence of the Axiom of Choice. Indeed, in the same manner as in the preceding illustration we have the following result: If $C$ is a nonempty compact convex subset of a Banach space and if $f: C \rightarrow C$ is a continuous operator, then the equation (Fp) has a solution on $C$. Thus, we have and Schauder's theorem as a consequence of the fact (b) in Theorem 1, i.e., as a consequence of the Axiom of Choice.

Illustration 5. (Schauder's problem). The most famous of many problems in nonlinear analysis is Schauder's problem (Scottish book, problem 54) of the following form, that if $C$ is a nonempty convex compact subset of a linear topological space does every continuous mapping $f: C \rightarrow C$ has a fixed point? The answer we give in [23] is yes.

Theorem 12 (Answer is yes for Schauder's problem). Let $C$ be a nonempty convex compact subset of a linear topological space $X$ and suppose $T: C \rightarrow$ $C$ is a continuous mapping. Then $T$ has a fixed point in $C$. 
The proof of this fact may be found in [23] by the aid of the Axiom of Choice, i.e., of Zorn's lemma. On the other hand, a proof that solution of Schauder's problem as Theorem 12 is a consequence of Lemma of Equation Points we can give in the same manner as the proof of Brouwer's theorem.

In this case also, de facto, we give that: if $C$ is a nonempty convex compact subset of a linear topological space and if $f: C \rightarrow C$ is a continuous mapping, then the equation (Fp) has a solution on $C$.

Illustration 6. (Caristi's theorem). In $1976 \mathrm{~J}$. Caristi investigated a mapping $f$ on a complete metric space $(X, \rho)$ that satisfies the following condition: there exists a lower semicontinuous function $G: X \rightarrow \mathbb{R}_{+}^{0}:=$ $[0,+\infty)$ such that

$$
\rho[x, f(x)] \leq G(x)-G(f(x)) \quad \text { for every } \quad x \in X,
$$

and showed that such mappings have a fixed point in space $X$. In this sense, define a relation $\preccurlyeq G, \rho$ on $X$ by the following condition in the form as:

$$
a \preccurlyeq \rho_{, G} b \quad \text { if and only if } \rho[a, b] \leq G(a)-G(b) \text {; }
$$

it is verify that $\preccurlyeq \rho, G$ is a partial ordering (asymmetric and transitive relation) in $X$. Via this ordering Kirk [Colloq. Math., 36 (1976), 81-86] give fact that Zorn's lemma implies Caristi's theorem.

On the other hand, a different proof that Caristi's theorem is a consequence of Lemma of Equation Points, i.e., of the Axiom of Choice we can give in the same manner as the proof of Brouwer's theorem via ordering $\preccurlyeq G, \rho$. For a different proof for this fact see: Browder [Seminar of Fixed Point Theory and its Appl., Dalhousie University, June 1975, 23-27].

Illustration 7. (Bounds roots of equations). We notice that, by the application of Lemma 2 by Tasković [23] (p. 241) one can simultaneously obtain the upper and lower bounds of the roots of the following equation in the form

$$
\begin{array}{r}
x^{n}=a_{1} x^{n-1}+a_{2} x^{n-2}+\cdots+a_{n} \\
\left(a_{1}+\cdots+a_{n}>0 ; \quad a_{i} \geq 0(i=1, \ldots, n)\right) .
\end{array}
$$

Then, by Minimax Principle in Tasković [23] (p. 245), as an immediate consequence we obtain the following statement for solutions of algebraic equations of the preceding form.

Theorem 13 (Taskovic $[23]$ ). A point $\xi \in \mathbb{R}_{+}:=(0,+\infty)$ is the root of the equation (AE) if and only if the following equality holds

$$
\begin{aligned}
\xi: & =\max _{\lambda_{2}, \ldots, \lambda_{n} \in \mathbb{R}_{+}} \min \left\{\lambda_{2}, \ldots, \lambda_{n}, a_{1}+\frac{a_{2}}{\lambda_{2}}+\cdots+\frac{a_{n}}{\left.\lambda_{n}^{n-1}\right\}=}\right. \\
& =\min _{\lambda_{2}, \ldots, \lambda_{n} \in \mathbb{R}_{+}} \max \left\{\lambda_{2}, \ldots, \lambda_{n}, a_{1}+\frac{a_{2}}{\lambda_{2}}+\cdots+\frac{a_{n}}{\lambda_{n}^{n-1}}\right\} .
\end{aligned}
$$


We notice that, if $0<a_{1}+\cdots+a_{n}<1$, then a root $\xi$ of the equation (AE) of the form (Ro) lie in the open interval $(0,1)$, i.e., $\xi \in(0,1)$, such that

$$
\begin{aligned}
\xi & =\max _{\lambda_{2}, \ldots, \lambda_{n} \in(0,1)} \min \left\{\lambda_{2}, \ldots, \lambda_{n}, a_{1}+\frac{a_{2}}{\lambda_{2}}+\cdots+\frac{a_{n}}{\left.\lambda_{n}^{n-1}\right\}=}\right. \\
& =\min _{\lambda_{2}, \ldots, \lambda_{n} \in(0,1)} \max \left\{\lambda_{2}, \ldots, \lambda_{n}, a_{1}+\frac{a_{2}}{\lambda_{2}}+\cdots+\frac{a_{n}}{\lambda_{n}^{n-1}}\right\} .
\end{aligned}
$$

We notice that this result is, de facto, also a consequence of the Axiom of Choice in the existence sense as a direct consequence of the Axiom of Choice, i.e., of Lemma of Equation Points.

Indeed, for $P:=[0,+\infty]$ with the ordinary ordering $\leq$ we have that $P$ is an inductive set and that in this case for function $f: P \rightarrow P$ defined by $f(x)=a_{1}+a_{2} / x+\cdots+a_{n} / x^{n-1}$ we have that condition (M) of (b) in Theorem 1 trivially holds in the form as

$$
x \leq \max \left\{x, a_{1}+\frac{a_{2}}{x}+\cdots+\frac{a_{n}}{x^{n-1}}\right\} \quad \text { for all } \quad x \in P ;
$$

hence, applying the statement (b) of Theorme 1 (i.e., Axiom of Choice) we obtain that the equation (AE) has a solution on $P$.

Annotation. We notice that the preceding result in above illustration is foundation on the fact that the equation in the form as

$$
\max \left\{x, a_{1}+\frac{a_{2}}{x}+\cdots+\frac{a_{n}}{x^{n-1}}\right\}=x
$$

has at least one solution on the totally ordered set $P:=[0,+\infty]$ because $x \mapsto x$ and $x \mapsto f(x)$ are continuous functions on $P$.

Illustration 8. (Further equivalents Axiom of Choice). In connection with preceding, let $(X, \rho)$ be a metric space and $G: X \rightarrow \mathbb{R}_{+}^{0}:=[0,+\infty)$ be a given function. Define a relation $\preccurlyeq \rho, G$ on $X$ as Brøndsted ordering by the following condition:

$$
a \preccurlyeq \rho, G b \quad \text { if and only if } \rho[a, b] \leq G(a)-G(b) \text { for all } a, b \in X .
$$

On the other hand, define a relation $\preccurlyeq_{G, \rho}$ on the metric space $X$ as Tasković ordering by the following condition:

$$
a \preccurlyeq G, \rho b \text { if and only if } \rho[a, b] \leq G(b)-G(a)
$$

for all $a, b, \in X$.

We notice that the ordering (B) is not dually, in comparable, with the ordering (Ta)!

In further, as immediate applications of the preceding Theorems 1 and 2 we have the following results.

In this sense as an immediate consequence of Theorem 2 we obtain by [23] the following result. 
Theorem 14 (Analytic Principles of Choice, Tasković in 1988). Let $P$ be $a$ partially ordered set with an ordered $\preccurlyeq$. Then the following statements are equivalent:

(a) (Statement (c) of Theorem 2). If $P$ is an inductive set and $f: P \rightarrow$ $P$ such that

$$
x \preccurlyeq f^{2}(x) \text { for all } x \in \operatorname{Sub} f(P),
$$

then $f$ has a fixed apex.

(b) (Tasković in 1993). If $(X, \rho)$ is a complete metric space and $G$ : $X \rightarrow \mathbb{R}_{+}^{0}$ is a lower semicontinuous function, then in the Brøndsted ordering every $f: X \rightarrow X$ satisfying $x \preccurlyeq \rho, G f^{2}(x)$ for every $x \in X$ has a fixed apex.

(c) (Tasković in 1993). If $(X, \rho)$ is a complete metric space and $G$ : $X \rightarrow \mathbb{R}$ is a bounded above and upper semicontinuous function, then in the ordering $\preccurlyeq_{G, \rho}$ every $f: X \rightarrow X$ satisfying $x \preccurlyeq G, \rho f^{2}(x)$ for every $x \in X$ has a fixed apex.

Short proof. Define relations $\preccurlyeq \rho, G$ and $\preccurlyeq G, \rho$ on $X$ by (B) and (Ta), and applying Theorem 1 in two direction, directly, we obtain this statement.

On the other hand, in the preceding sense, as an immediate consequence of Theorem 1 we obtain by [23] the following result.

Theorem 15 (Analytic forms of Axiom of Choice, Tasković in 1988)). Let $P$ be a partially ordered set with an ordered $\preccurlyeq$. Then the following statements are equivalent:

(d) (Statement (c) of Theorem 1). If $P$ is an inductive set and $f: P \rightarrow$ $P$ such that

$$
x \preccurlyeq f(x) \text { for all } x \in \operatorname{Sub} f(P),
$$

then $f$ has a fixed point.

(e) (Caristi in 1976). If $(X, \rho)$ is a complete metric space and $G: X \rightarrow$ $\mathbb{R}_{+}^{0}$ is a lower semicontinuous function, then in the Brøndsted ordering every $f: X \rightarrow X$ satisfying $x \preccurlyeq \rho, G f(x)$ for every $x \in X$ has a fixed point.

(h) (Tasković in 1986 and in 1988). If $(X, \rho)$ is a complete metric space and $G: X \rightarrow \mathbb{R}$ is a bounded above and upper semicontinuous function, then in the ordering $\preccurlyeq_{G, \rho}$ every $f: X \rightarrow X$ satisfying $x \preccurlyeq G, \rho f(x)$ for every $x \in X$ has a fixed point.

Short proof. Define relations $\preccurlyeq \rho, G$ and $\preccurlyeq G, \rho$ on $X$ by (B) and (Ta), and applying Theorem 1 in two direction, we obtain immediately this statement.

Illustration 9. (Banach's theorem). The notion of order (and the notion of completeness) have each led to fixed point theorems. We now obtain Banach contraction theorem as a consequence of the Axiom of Choice. In 
this sense let $(X, \rho)$ be a metric space, $T: X \rightarrow X$, and define a relation $\preccurlyeq$ on $X$ by

$$
x \preccurlyeq y \quad \text { if and only if } \rho[x, y] \leq G(x)-G(y)
$$

for all $x, y \in X$, where $G(x)=(1-\alpha)^{-1} \rho[x, T x]$ and $\alpha \in[0,1)$. It is easy to verify that $\preccurlyeq$ is a partial ordering in $X$. Thus $\preccurlyeq:=\preccurlyeq \rho, G$ and we have (as in Illustration 6) that Banach's theorem is a consequence of Lemma of Equation Points, i.e., of the Axiom of Choice.

Annotation. In connection with the preceding facts from [23] see and papers: $\mathrm{K} \mathrm{ir} \mathrm{k}$ in 1976, H. Höft and P. Howard in 1994, A bian in 1985, Turinici in 1984, Brunner in 1987, Makowski and Wiśniewski in 1969, Baker in 1964, Mańka in 1988, Kenyon in 1963, Dj. Kurepa in 1952, and Brøndsted in 1979.

Illustration 10. (General expansion mappings). Let $X$ be a topological space, $T: X \rightarrow X$, and let $A: X \times X \rightarrow \mathbb{R}_{+}^{0}:=[0,+\infty)$. We shall introduce the concept of CS-convergence in a space $X$; i.e., a topological space $X$ satisfies the condition of CS-convergence iff $\left\{x_{n}\right\}_{n \in \mathbb{N}}$ is a sequence in $X$ and $A\left(x_{n}, T x_{n}\right) \rightarrow 0(n \rightarrow \infty)$ implies that $\left\{x_{n}\right\}_{n \in \mathbb{N}}$ has a convergent subsequence.

Also, we shall introduce the concept of invariant property for space $X$; i.e., a topological space $X$ satisfies the condition of invariant property if there is a nonempty subset $A$ of $X$ such that $T(A)=A$. Obviously, if $T: X \rightarrow X$ is an onto mapping, then $X$ is with the invariant property for $A=X$. Also, if $T: X \rightarrow X$ continuous on a compact space $X$, then $X$ has the invariant property.

Lemma 6. Let the mapping $\varphi: \mathbb{R}_{+} \rightarrow \mathbb{R}_{+}:=(0,+\infty)$ have the following properties in the following form as

$$
\varphi(t)>t \quad \text { and } \quad \liminf _{z \rightarrow t-0} \varphi(z)>t
$$

for every $t \in \mathbb{R}_{+}$. If the sequence $\left\{x_{n}\right\}_{n \in \mathbb{N}}$ of real positive numbers satisfies the inequality of the form as

$$
x_{n} \geq \varphi\left(x_{n+1}\right) \quad \text { for all } n \in \mathbb{N},
$$

then it converges to zero. The velocity of this convergence is not necessarily geometric.

Proof. Since $\left\{x_{n}\right\}_{n \in \mathbb{N}}$ is a nonincreasing bounded sequence in $\mathbb{R}_{+}$, there is a $t \geq 0$ such that $x_{n} \rightarrow t(n \rightarrow \infty)$. We claim that $t=0$. If $t>0$, then

$$
t=\liminf _{n \rightarrow \infty} x_{n} \geq \liminf _{n \rightarrow \infty} \varphi\left(x_{n+1}\right) \geq \liminf _{z \rightarrow t-0} \varphi(z)>t,
$$

which is a contradiction. Consequently $t=0$ and so $x_{n} \rightarrow 0(n \rightarrow \infty)$. The proof is complete.

We are now in a position to formulate the following general statements from: [23]. Also see: [19]. 
Theorem 16 (General expansion). Let $T$ be a mapping of a topological space $X$ into itself, where $X$ with the invariant property and with the condition of CS-convergence. If there is a mapping $\varphi: \mathbb{R}_{+}^{0} \rightarrow \mathbb{R}_{+}^{0}$ such that the condition $(\varphi)$ holds and

$$
A(T x, T y) \geq \varphi(A(x, y)) \text { for all } x, y \in X,
$$

where $A: X \times X \rightarrow \mathbb{R}_{+}^{0}, x \mapsto A(x, T x)$ is lower semicontinuous and $A(a, b)=0$ implies $a=b$, then $T$ has a unique fixed point in $X$.

As immediate consequences of the preceding statement we obtain results in 1984 of Wang, Gao, Li and Iséki and in 1992 of Daffer and Kaneko's.

Proof of Theorem 16. (Application of Axiom of Choice). Since $X$ is with invariant property, there exists a nonempty subset $A$ of $X$ such that $T(A)=$ $A$. Then the set of the form $A_{x}:=T^{-1}(\{x\}) \subset A$ is a nonempty subset of $A$ for every $x \in A$. If $g \mid A$ is a function of choice, then there is a sequence $\left\{a_{n}\right\}_{n \in \mathbb{N}}$ in $X$ defined by $a_{n+1}=g\left(A_{a_{n}}\right)$ for $n \in \mathbb{N}$, where $a_{1} \in A$ is an arbitrary point. Thus we obtain that $a_{n}=T\left(a_{n+1}\right)$ for all $n \in \mathbb{N}$, in $X$. From (An) we have

$$
A\left(a_{n}, a_{n+1}\right)=A\left(T\left(a_{n+1}\right), T\left(a_{n+2}\right)\right) \geq \varphi\left(A\left(a_{n+1}, a_{n+2}\right)\right)
$$

for all $n \in \mathbb{N}$. Applying Lemma 6 to the sequence $\left\{A\left(a_{n}, a_{n+1}\right)\right\}_{n \in \mathbb{N}}$, we obtain $A\left(a_{n}, a_{n+1}\right) \rightarrow 0(n \rightarrow \infty)$. This implies (from CS-convergence) that the sequence $\left\{a_{n}\right\}_{n \in \mathbb{N}}$ contains a convergent subsequence $\left\{a_{n(k)}\right\}_{k \in \mathbb{N}}$ with limit $\xi \in X$. Since $x \mapsto A(x, T x)$ is lower semicontinuous at $\xi$, we obtain

$$
A(\xi, T \xi) \leq \liminf A\left(a_{n(k)}, a_{n(k)-1}\right)=\liminf A\left(a_{n}, a_{n-1}\right)=0,
$$

i.e., $T \xi=\xi$. We complete the proof by showing that $T$ can have at most one fixed point. In fact, if $\xi \neq \eta$ were two fixed points, then $A(\xi, \eta)=$ $A(T \xi, T \eta) \geq \varphi(A(\xi, \eta))>A(\xi, \eta)$, which is a contradiction. The proof is complete.

\section{REFERENCES}

[1] A. Abian, A fixed point theorem equivalent to the axiom of choice, Abstracts Amer. Math. Soc., abstract no. 388, 4 (1983).

[2] H. Amann, Order structures and fixed points, Ruhr-Universität, Bochum, (mimeographed lecture notes), 1977.

[3] G. Birkhoff, Lattice theory, Amer. Math. Soc. Colloq. Publ., vol. 25, New York, 1948.

[4] N. Bourbaki, Sur le theoreme de Zorn, Archiv der Math. 2 (1950), 434-437.

[5] J. Caristi, Fixed point theorems for mappings satisfying inwardness conditions, Trans. Amer. Math. Soc., 215 (1976), 241-251.

[6] K. Gödel, The consistency of the continuum hypothesis, Ann. of Math. Stud., vol. 3, Princeton Univ. Press, Princeton, NJ, 1940, pp. 66.

[7] P. Howard, and J.E. Rubin, Consequences of the Axiom of Choice, American Math. Society, Vol. 59, 1998. Mathematical Surveys and Monographs, 432 pages. 
[8] T.J. Jech, The Axiom of Choice, North-Holland, Amsterdam, 1973.

[9] J. Klimeš, Fixed edge theorems for complete lattices, Arch. Math. 17 (1981), 227-234.

[10] C. Kuratowski, Une methode d'elimination des nombers transfinies raisonnements mathematiques, Fund. Math. 3 (1922), 76-108.

[11] G. Kurepa, Sur la relation d'inclusion et l'axiome de choix de Zermelo, Bull. Soc. Math. France 80 (1952), 225-232.

[12] A. Mostowski, On the principle of dependent choices, Fund. Math. 35 (1948), 127-130.

[13] G.H. Moore, Zermelo's Axiom of Choice, its origins, development and influence, Springer-Verlag, New York, Heidelberg, and Berlin, pp. 410.

[14] H. Rubin, On a problem of Kurepa concerning the axiom of choice, Notices Amer. Math. Soc. 5 (1958), no. 378.

[15] H. Rubin and J. Rubin, Equivalents of the Axiom of Choice, North-Holland, Amsterdam and London, 1970, p. 134.

[16] H. Rubin and J. Rubin, Equivalents of the Axiom of Choice II, North-Holland, Amsterdam, New York and Oxford, 1985, p. 322.

[17] H. Rubin and J. Rubin, Some new forms of the axiom of choice, Notices Amer. Math. Soc. 7 (1960), no. 380.

[18] A. Tarski, A lattice theoretical fixed point theorem and its applications, Pacific J. Math. 5 (1955), 285-309.

[19] M.R. Tasković, Banach's mappings of fixed points on spaces and ordered sets, Thesis, Math. Balkanica 8 (1978), 150 pages.

[20] M.R. Tasković, Characterizations of inductive posets with applications, Proc. Amer. Math. Soc. 104 (1988), 650-660.

[21] M.R. Tasković, On an equivalent of the axiom of choice and its applications, Math. Japonica 31 (1986), 979-991.

[22] M.R. Tasković, The axiom of choice, fixed point theorems, and inductive ordered sets, Proc. Amer. Math. Soc., 116 (1992), 897-904.

[23] M.R. Tasković, Theory of transversal point, spaces, and forks, Monographs of a new mathematical theory, VIZ-Beograd 2005, (in Serbian), 1054 pages. English summary: 1001-1022.

[24] E. Zermelo, Neuer Beweis fur die Moglichkeit einer Wohlordnung, Math. Ann. 65 (1908), 107-128; translated in van Heijenoort 1967, 183-198.

[25] Max Zorn, A remark on method in transfinite algebra, Bull. Amer. Math. Soc. 41 (1935), 667-670.

Milan R. Tasković

FACULTY OF MATHEMATiCs

11000 Belgrade, P.O. Box 550

SERBIA

Home Address:

Milan R. Tasković

Nehruova 236

11070 BELGRADE

SERBIA

E-mail address: andreja@predrag.us 